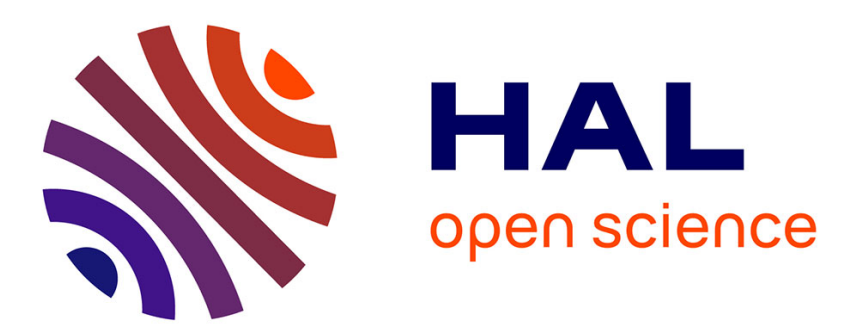

\title{
The propagation of low-viscosity fingers into fluid-filled branching networks
}

\author{
Charles N. Baroud, Sedina Tsikata, M. Heil
}

\section{To cite this version:}

Charles N. Baroud, Sedina Tsikata, M. Heil. The propagation of low-viscosity fingers into fluid-filled branching networks. Journal of Fluid Mechanics, 2006, 546 (january), pp.285-294. $10.1017 / \mathrm{s} 0022112005007287$. hal-01023373

\section{HAL Id: hal-01023373}

https: / hal-polytechnique.archives-ouvertes.fr/hal-01023373

Submitted on 28 Jul 2014

HAL is a multi-disciplinary open access archive for the deposit and dissemination of scientific research documents, whether they are published or not. The documents may come from teaching and research institutions in France or abroad, or from public or private research centers.
L'archive ouverte pluridisciplinaire HAL, est destinée au dépôt et à la diffusion de documents scientifiques de niveau recherche, publiés ou non, émanant des établissements d'enseignement et de recherche français ou étrangers, des laboratoires publics ou privés. 


\title{
The propagation of low-viscosity fingers into fluid-filled branching networks
}

\author{
By CHARLES N. BAROUD ${ }^{1}$, SEDINA TSIKATA \\ AND MATTHIAS HEIL ${ }^{2}$ \\ ${ }^{1}$ Laboratoire d'Hydrodynamique (LadHyX), Ecole Polytechnique, 91128-F Palaiseau Cedex, France \\ ${ }^{2}$ School of Mathematics, University of Manchester, Manchester, M13 9PL UK
}

(Received 12 January 2005 and in revised form 20 June 2005)

We consider the motion of a finger of low-viscosity fluid as it propagates into a branching network of fluid-filled microchannels - a scenario that arises in many applications, such as microfluidics, biofluid mechanics (e.g. pulmonary airway reopening) and the flow in porous media. We perform experiments to investigate the behaviour of the finger as it reaches a single bifurcation and determine under what conditions the finger branches symmetrically. We find that if the daughter tubes have open ends, the finger branches asymmetrically and will therefore tend to reopen a single path through the branching network. Conversely, if the daughter tubes terminate in elastic chambers, which provide a lumped representation of the airway wall elasticity in the airway reopening problem, the branching is found to be symmetric for sufficiently small propagation speeds. A mathematical model is developed to explain the experimentally observed behaviour.

\section{Introduction}

Flows in which a finger of low-viscosity fluid propagates into a tube and displaces another fluid of larger viscosity are of relevance in many applications, such as oil extraction (Homsy 1987), microfluidic devices for droplet transport (Link et al. 2004) and pulmonary biomechanics (Gaver, Samsel \& Solway 1990).

For instance, many respiratory diseases, such as respiratory distress syndrome, may cause the occlusion of the pulmonary airways with viscous fluid. Occluded airways are believed to be reopened by a propagating air finger, in a process that involves a complicated interaction between a viscous free-surface flow and the deformation of the elastic airway wall. The mechanics of airway reopening in an individual airway have been investigated by many authors (e.g. Gaver et al. 1990; Hazel \& Heil 2003), but these studies ignore the fact that the pulmonary airways branch frequently. This raises the question of whether the propagating air finger will reopen the entire pulmonary tree or simply follow a single path, keeping most of the lung occluded. (Cassidy, Gavriely \& Grotberg (2001) performed experiments to determine how short liquid plugs propagate through rigid bifurcations.) The question is also of relevance in 'gas embolotherapy', a novel technique aimed at starving tumour cells of their blood supply (see Calderon \& Bull 2004).

Similar questions arise in microfluidic technology. Many microfluidic devices are designed to transport samples of fluid through complicated networks of channels in order to perform chemical or bio-chemical tests (Song, Tice \& Ismagilov 2003). The use of drops as vehicles for the transport offers many advantages, such as 

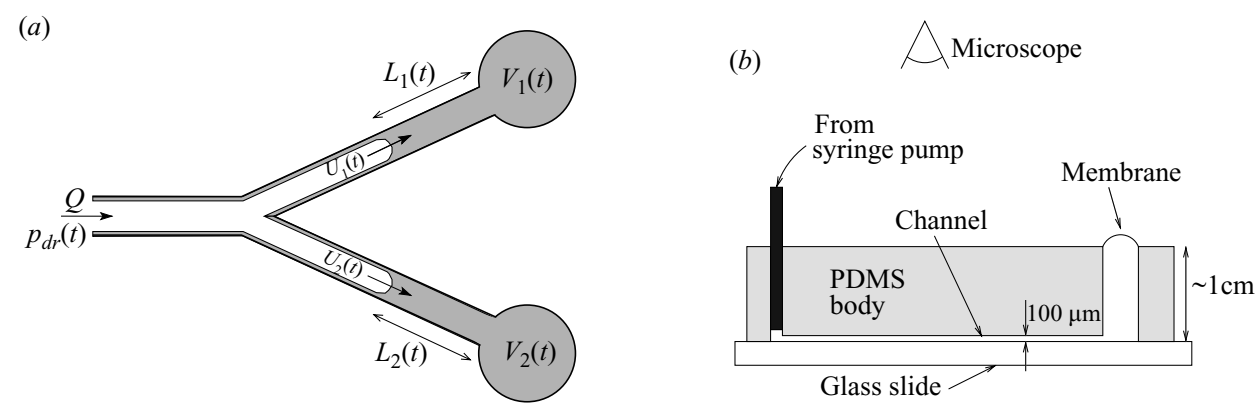

Figure 1. (a) Model problem: low-viscosity fluid is injected into a branching tube. The propagating fingers displace some of the more viscous fluid that initially filled the tube; the remaining viscous fluid is deposited on the tube walls. The two daughter tubes are terminated by large elastic chambers. (b) Sketch of the experimental set-up (not to scale). The total volume of fluid contained in the channel is approximately $0.5 \mu \mathrm{l}$ while the volume in the end chambers is approximately $50 \mu 1$.

the control of dispersion and wall contact, as well as the possibility of activating chemical reactions at controlled locations, e.g. through droplet merging. Other uses of bubbles and drops in microfluidics include the production of controlled microemulsions through the breakup of long bubbles as they advance through a branching network of microchannels (Link et al. 2004). It is therefore important to understand the dynamics of the drop transport in order to predict the path and the breakup of individual drops.

Here, we study these questions at the level of a single bifurcation and consider the model problem shown in figure 1 $(a)$. A finger of low-viscosity fluid propagates along a parent tube which branches into two daughter tubes which have identical cross-sections. The finger is driven by the injection of fluid at a constant flow rate, $Q$, and displaces a viscous fluid of viscosity $\mu$ which is much larger than the viscosity of the finger. We denote the surface tension between the two fluids by $\sigma$. The daughter tubes terminate in two large elastic chambers which provide a lumped representation of the wall elasticity in the pulmonary airways. We wish to establish the conditions under which the finger continues to propagate symmetrically along the two daughter tubes once it has passed the bifurcation.

\section{Experimental set-up}

We performed experiments using the set-up sketched in figure 1(b). A Y-shaped channel of rectangular cross-section $(W \times H=200 \mu \mathrm{m} \times 100 \mu \mathrm{m})$ was fabricated using standard microfluidic soft lithography methods (Quake \& Scherer 2000). A mould was made by etching a $100 \mu \mathrm{m}$ layer of photoresist on the surface of a silicon wafer. The mould was then covered with a thick layer of liquid poly(dimethylsiloxane) (PDMS) which was allowed to solidify before we created cylindrical chambers at the ends of the three branches: a thin hole at the end of the parent branch for the fluid injection, and two larger end chambers (holes with $1.6 \mathrm{~mm}$ radii) at the ends of the two daughter channels. The large end chambers were sealed with thin elastic membranes of thickness $h=50 \mu \mathrm{m}$, also made of PDMS (Young's modulus $E \simeq 9 \times 10^{5} \mathrm{~N} \mathrm{~m}^{-2}$ and Poisson's ratio $v=0.5$ ). Finally, the PDMS block was bonded onto a glass microscope slide. 


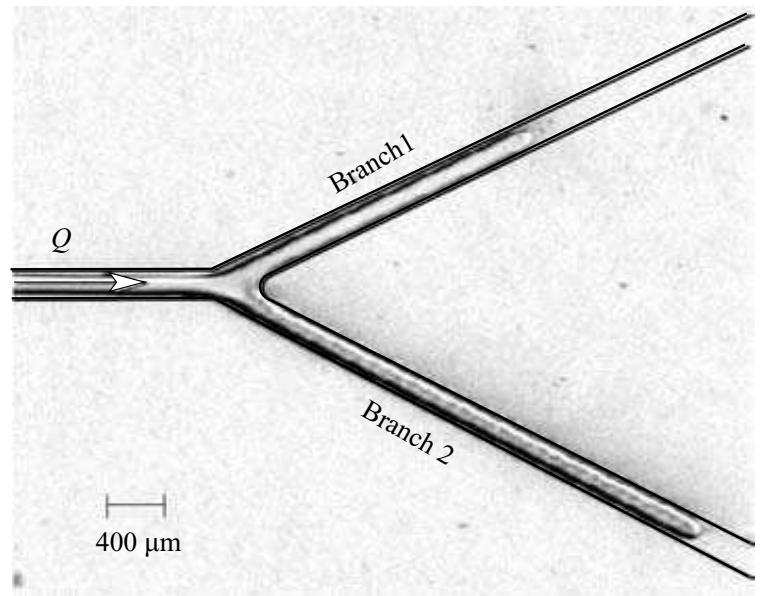

FIGURE 2. Snapshot of the branching finger. The positions of the two tips are recorded over a sequence of successive images, allowing the velocity of the fingers to be determined.

The experiments were conducted by first filling the channels with silicone oil (viscosity $\mu=100 \mathrm{cP}$ ) until the end membranes were taut. PDMS is permeable to gas, therefore the air that initially filled the channels could escape through the thin membranes when the silicone oil was injected. After the membranes were slightly prestretched, a finger of perfluorodecalin (PFD; viscosity $\mu_{P F D}=2.9 \mathrm{cP}$ ) was injected. This was done using a syringe pump with a $100 \mu$ l glass syringe, at flow rates in the range $1-60 \mathrm{nl} \mathrm{s}^{-1}$, resulting in finger velocities of $50-3000 \mu \mathrm{m} \mathrm{s}^{-1}$ in the main branch. The choice of fluids was guided by the requirements that the two fluids be incompressible and immiscible, and that they wet the PDMS and the glass surfaces. The surface tension between the two fluids is approximately $\sigma=50 \mathrm{mN} \mathrm{m}^{-1}$.

Image sequences of the advancing fingers were taken through a stereo-microscope at $\times 7.11$ magnification, using a digital camera at $2000 \times 2000$ pixel resolution. A typical image is shown in figure 2 . We tracked the position of the advancing fingers in the two daughter tubes over a sequence of images to determine the speed of the two finger tips.

\section{Experimental results}

Figure 3 shows the evolution of the finger velocities in the two daughter tubes for fingers that are driven by the injection of fluid at two different flow rates. At low flow rates the fingers propagate at approximately the same speed, resulting in an approximately symmetric branching pattern. Conversely, at higher flow rates, any small initial difference in the finger speeds increases rapidly as the fingers propagate into the daughter tubes, resulting in strongly asymmetric branching. We observe that in both cases the sum of the two finger speeds remains constant within experimental error. Experiments conducted in open-ended channels, i.e. without the elastic membranes, always displayed strongly asymmetric propagation, regardless of the driving flow rate. Finally, we note that the initial filling of the viscous oil into an air-filled channel always occurred in a symmetric manner, both with and without the elastic membranes. This is consistent with the experiments of Cassidy et al. (2001) who found that finite-length plugs of oil branched symmetrically when flowing through a symmetric air-filled bifurcation. 

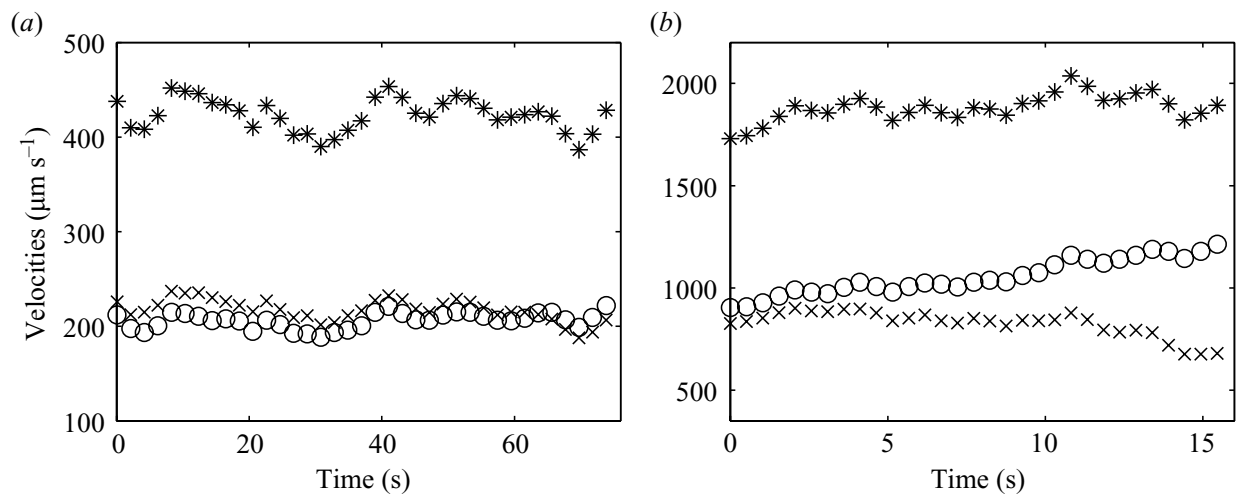

FIGURE 3. Time series of the velocity of the advancing fingers in channels $1(O)$ and $2(x)$ as a function of time, for two different forcing flow rates. The sum of the two velocities $(*)$ remains approximately constant.

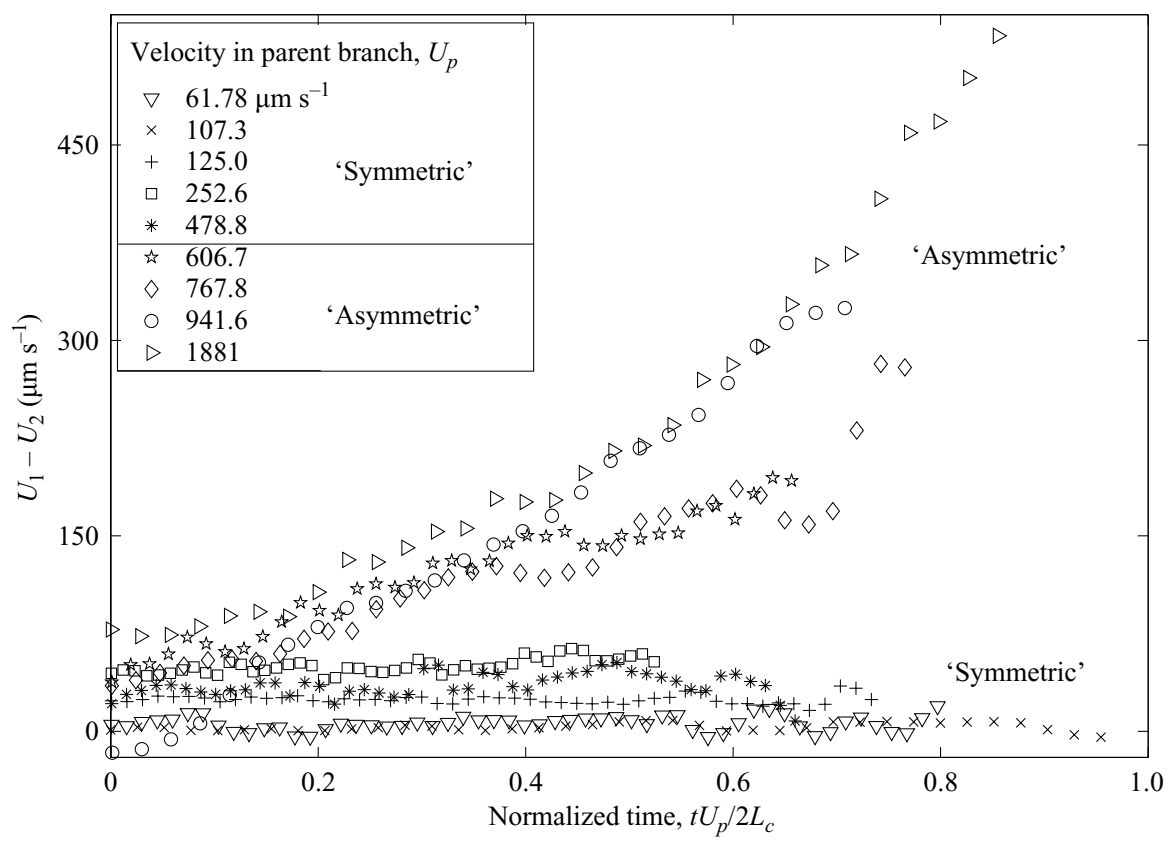

FIGURE 4. Evolution of the velocity difference for the fingers in the two daughter branches. At low flow rates, the velocity differences remain constant. When the velocity in the parent branch exceeds a threshold, the difference in the velocities increases rapidly. We refer to the two regimes as 'symmetric' and 'asymmetric', respectively.

Results for channels with elastic chambers are presented in figure 4 where the symmetry of the branching is characterized by plotting the difference between the finger velocities as a function of the 'normalized distance' $t U_{p} / 2 L_{c}$, where $L_{c}=1.7 \mathrm{~cm}$ is the length of the daugther channels. This ordinate facilitates a direct comparison between the different experiments whose duration varies significantly.

At small flow rates, the difference in finger speeds remains approximately constant. When the velocity in the parent channel becomes larger than a threshold, the velocity difference increases rapidly as the fingers propagate along the daughter branches - the 
experiment ends when the 'faster' finger reaches the downstream end of its daughter tube. For a given microchannel, the faster finger is always associated with the same branch, suggesting that small imperfections in the fabrication process produce the initial perturbation. These initial perturbations are strongly amplified at high flow rates but remain almost constant if the flow rate is below a threshold. For simplicity, we will refer to the two regimes as 'asymmetric' and 'symmetric', respectively.

The signature of the asymmetric regime is also observed when the low-viscosity finger is not continuous, but made up of discrete drops. These drops may be produced by a splitting of the PFD finger at the entrance of the microchannel, for example if the connecting tube is lined with silicone oil. As the train of drops reaches the bifurcation, each is split into two 'daughter droplets' which travel down the daughter channels. The length ratio of the two daughter drops depends on the relative velocity in the two branches. In the symmetric case, the size ratio remains constant as successive drops arrive at the bifurcation, indicating a constant velocity difference. In the asymmetric case, the velocity difference increases and so does the length ratio of the divided daughter drops. This ratio may eventually diverge as the fast finger approaches the end of the channel and the flow in the other branch slows to a halt.

\section{Analysis}

We will now develop a simple mathematical model to explain the experimental observations. Since the finger is driven by the injection of fluid at a constant flow rate $Q$, the pressure, $p_{d r}(t)$, required to drive the finger at this rate varies with time. We denote the lengths of the fluid-filled parts of the daughter tubes ahead of the finger tips by $L_{i}(t)(i=1,2)$ and assume that the daughter tubes have the same cross-sectional area, $A$.

As the fingers propagate, they displace most of the viscous fluid that initially filled the tube; far behind the finger tips, a thin stationary film of the more viscous fluid is deposited on the tube walls (see Taylor 1961). We denote the flow rate in daughter tube $i$ by $Q_{i}=U_{i} A_{i}$, where $A_{i}$ is the cross-sectional area occupied by the finger which propagates with speed $U_{i}$. In the absence of inertial and gravitational effects, a reasonable approximation for microfluidic devices, we have $A_{i}=A \alpha\left(U_{i}\right)$, where the function $\alpha\left(U_{i}\right)$ has been determined for many tube shapes (e.g. Bretherton (1961) and Reinelt \& Saffman (1985) for circular tubes; Wong, Radke \& Morris (1995) for polygonal tubes; and Hazel \& Heil (2002) for tubes of elliptical and rectangular cross-section - we will refer to these references collectively as 'R'). Conservation of mass requires that $Q_{1}+Q_{2}=Q$.

In each of the daughter tubes, the pressure drop across the occluded section has three components: (i) the Poiseuille pressure drop ahead of the finger tip, $\Delta p_{\text {pois }}=$ $\mu \mathscr{R} L_{i}(t) Q_{i}(t)$, where the flow resistance $\mathscr{R}$ depends on the tube's cross-section. For instance, for a circular tube of radius $a$, we have $\mathscr{R}=8 /\left(\pi a^{4}\right)$; values for other cross-sections can be found in the literature (e.g. White 1991); (ii) the pressure drop across the curved tip of the finger, $\Delta p_{\text {tip }}=\mathscr{C}\left(U_{i}\right)$, where the function $\mathscr{C}\left(U_{i}\right)$ for many tube shapes is available from the references $\mathrm{R}$ (we note that $\Delta p_{\text {tip }}$ includes capillary and viscous contributions); (iii) the pressure $p_{\text {elast }}$ in the chambers at the end of the daughter tubes. Provided the membranes that close the (otherwise rigid) chambers are elastic, $p_{\text {elast }}$ depends only on the chambers' instantaneous volume and we assume that $p_{\text {elast } i}(t)=k \int_{0}^{t} Q_{i}(\tau) \mathrm{d} \tau+p_{0 i}$, where $k$ is a constant and $p_{0 i}$ is the pressure in chamber $i$ at time $t=0$. 
Assuming that the viscous pressure drop in the fingers can be neglected, the fingers in both daughter tubes are subject to the same driving pressure $p_{d r}(t)$, and we have

$$
p_{d r}(t)=\mathscr{C}\left(U_{i}\right)+\mu \mathscr{R} L_{i}(t) Q_{i}(t)+k \int_{0}^{t} Q_{i}(\tau) \mathrm{d} \tau+p_{0 i} \quad \text { for } \quad i=1,2,
$$

where $Q_{i}(t)=A U_{i}(t) \alpha\left(U_{i}(t)\right)$. Differentiating the two equations in (4.1) with respect to $t$ yields

$$
\begin{aligned}
\frac{\mathrm{d} p_{d r}(t)}{\mathrm{d} t}= & \left.\frac{\mathrm{d} U_{i}(t)}{\mathrm{d} t} \frac{\mathrm{d} \mathscr{C}(U)}{\mathrm{d} U}\right|_{U=U_{i}(t)}+k A \alpha\left(U_{i}(t)\right) U_{i}(t)+\mu A \mathscr{R}\left(\frac{\mathrm{d} L_{i}(t)}{\mathrm{d} t} \alpha\left(U_{i}(t)\right) U_{i}(t)\right. \\
& \left.+\left.L_{i}(t) \frac{\mathrm{d} U_{i}(t)}{\mathrm{d} t} \frac{\mathrm{d} \alpha(U)}{\mathrm{d} U}\right|_{U=U_{i}(t)} U_{i}(t)+L_{i}(t) \alpha\left(U_{i}(t)\right) \frac{\mathrm{d} U_{i}(t)}{\mathrm{d} t}\right) \text { for } i=1,2 .
\end{aligned}
$$

Together with the two equations $U_{i}=-\mathrm{d} L_{i} / \mathrm{d} t$ (for $i=1,2$ ), this provides a system of four ordinary differential equations, augmented by the algebraic constraint $U_{1}(t) \alpha\left(U_{1}(t)\right)+U_{2}(t) \alpha\left(U_{2}(t)\right)=Q / A$. Thus, we have five equations for the five unknowns $p_{d r}, U_{1}, U_{2}, L_{1}$ and $L_{2}$. If $p_{01}=p_{02}$, these equations admit the symmetric solution $L_{i}(t)=l_{0}-\mathscr{U} t$, where the finger velocity $U_{i}=\mathscr{U}$ in both daughter tubes is given implicitly by

$$
\mathscr{U} \alpha(\mathscr{U})=\frac{1}{2} \frac{Q}{A} .
$$

We determine the stability of this solution by writing the velocities as $U_{i}(t)=\mathscr{U}+\epsilon \widehat{U}_{i}(t)$, where $\epsilon \ll 1$, with similar expansion for all other quantities. A straightforward linear stability analysis then yields the relations $\widehat{L}_{1}(t)+\widehat{L}_{2}(t)=0$ and $\mathrm{d} \widehat{p}_{d r} / \mathrm{d} t=0$ for the perturbed quantities. The velocity perturbations are governed by

$$
\int_{\widehat{U}_{i}(t=0)}^{\widehat{U}_{i}(t)} \frac{\mathrm{d} u}{u}=\int_{0}^{t} \frac{\mathscr{U} \mathscr{F}(\mathscr{U})}{\mathscr{G}(\mathscr{U}, \tau)} \mathrm{d} \tau
$$

where

$$
\mathscr{G}(\mathscr{U}, t)=\left(l_{0}-\mathscr{U} t\right)\left(\alpha(\mathscr{U})+\left.\frac{\mathrm{d} \alpha(U)}{\mathrm{d} U}\right|_{\mathscr{U}} \mathscr{U}\right)+\left.\frac{1}{\mu \mathscr{R} A} \frac{\mathrm{d} \mathscr{C}(U)}{\mathrm{d} U}\right|_{\mathscr{U}}
$$

and

$$
\mathscr{F}(\mathscr{U})=\alpha(\mathscr{U})\left(2-\frac{k}{\mu \mathscr{U} \mathscr{R}}\right)+\left.\mathscr{U} \frac{\mathrm{d} \alpha(U)}{\mathrm{d} U}\right|_{\mathscr{U}}\left(1-\frac{k}{\mu \mathscr{U} \mathscr{R}}\right) .
$$

If the right-hand side of (4.3) is negative, the perturbation velocities $\widehat{U}_{i}$ decay, indicating that the symmetrically branching solution is stable. To analyse equation (4.3), we first consider the simplifications $\alpha \equiv 1$ (ignoring the presence of the fluid film that the advancing fingers deposit on the channel walls) and $\mathscr{C} \equiv 0$ (ignoring the pressure jump over the air-liquid interface). In this case, (4.3) simplifies to

$$
\int_{\widehat{U}_{i}(t=0)}^{\widehat{U}_{i}(t)} \frac{\mathrm{d} u}{u}=\int_{0}^{t} \frac{2 \mathscr{U}-k /(\mu \mathscr{R})}{l_{0}-\mathscr{U} \tau} \mathrm{d} \tau .
$$

The denominator of the integrand on the right-hand side represents the instantaneous length of the fluid-filled part of the daughter tubes and is therefore always positive. Perturbations to the symmetrically branching finger will therefore grow if $\mathscr{U}$ exceeds the critical value $U_{c}=k /(2 \mu \mathscr{R})$. Hence at small velocities, when the flow resistance is dominated by the vessel stiffness, the finger will branch symmetrically. Conversely, at 


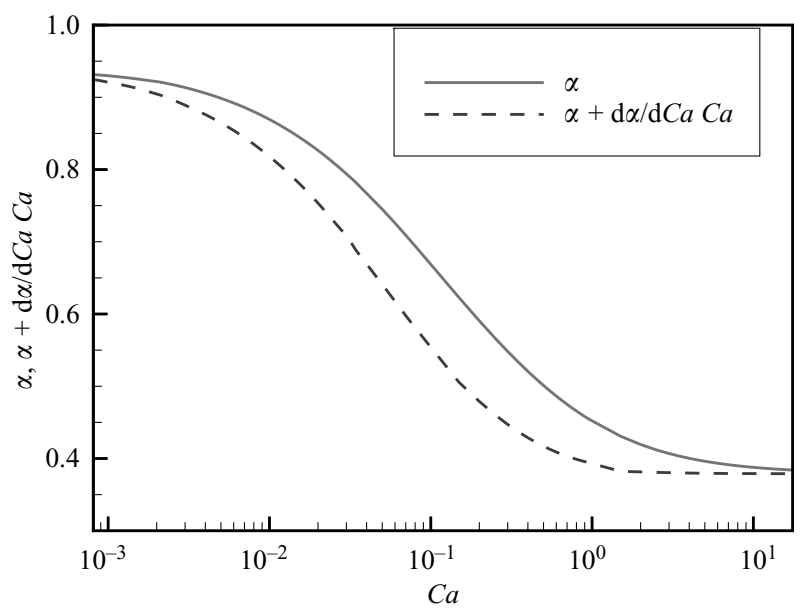

FIGURE 5. The fraction $\alpha$ of the tube's cross-section that is occupied by the propagating finger as a function of the capillary number $C a=\mu \mathscr{U} / \sigma$ for tubes with square cross-sections. Results for tubes of rectangular, circular and elliptical cross-sections are qualitatively similar.

large finger velocities, the resistance to the flow is dominated by the viscous losses and the propagating finger will tend to open a single path through the branching network.

Without the approximations used in the derivation of (4.6), we must determine the signs of various terms in (4.4) and (4.5). References $\mathrm{R}$ show that $\mathrm{d} \mathscr{C}(U) / \mathrm{d} U>0$ and, using Hazel \& Heil's (2002) computational results, we find that $\alpha+\left.\mathscr{U}(\mathrm{d} \alpha / \mathrm{d} U)\right|_{\mathscr{U}}>0$ (see figure 5 for the case of tubes with square cross-section; the curves for tubes with rectangular, circular and elliptical cross sections are qualitatively similar). Hence $\mathscr{G}(\mathscr{U}, t)>0$, implying that the growth or decay of the perturbations is determined by the sign of $\mathscr{F}$ which we rewrite in non-dimensional form as

$$
\mathscr{F}(C a)=\alpha(C a)\left(2-\frac{\mathscr{K}}{C a}\right)+C a \frac{\mathrm{d} \alpha(C a)}{\mathrm{d} C a}\left(1-\frac{\mathscr{K}}{C a}\right) .
$$

Here, $C a=\mu \mathscr{U} / \sigma$ is the capillary number based on the propagation speed of the symmetrically propagating fingers and the dimensionless parameter $\mathscr{K}=k /(\mathscr{R} \sigma)$ is a measure of the stiffness of the elastic end chambers.

Figure 6 shows plots of $\mathscr{F}(\mathrm{Ca})$ for square tubes and for a range of values of the parameter $\mathscr{K}$. Also shown (as dashed lines) are the approximations $\mathscr{F}(\mathrm{Ca}) \approx 2-$ $\mathscr{K} / C a$ which correspond to the simplifications $\alpha \equiv 1$ and $\mathscr{C} \equiv 0$ used in the derivation of (4.6). Figure 6 shows that the critical velocity $U_{c}$ obtained from the approximate analysis provides an excellent prediction for critical velocity at which the symmetrically branching solution becomes unstable. This is because the capillary pressure jump affects only the magnitude of the growth rate, but not its sign, therefore setting $\mathscr{C} \equiv 0$ does not affect the prediction for $U_{c}$. Furthermore, at small capillary number, the thickness of the fluid film that the propagating finger deposits on the channel walls is small, therefore the approximation $\alpha \equiv 1$ becomes more accurate as the capillary number is reduced. This explains why the discrepancy between the exact and the approximate solutions for $U_{c}$ decreases with $C a$. Both analyses show that if $k=0$, corresponding to the case when the end chambers are open to the atmosphere and 


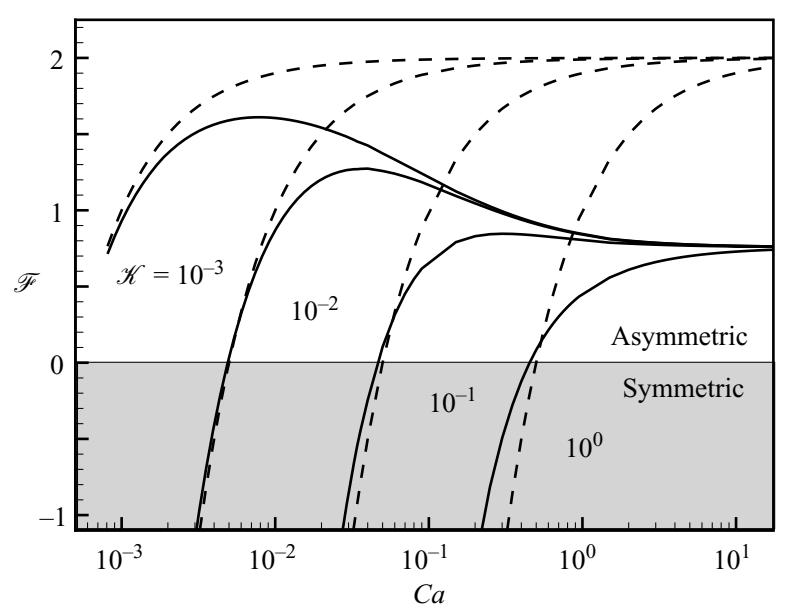

FIgURE 6. The function $\mathscr{F}(\mathrm{Ca})$ for tubes of square cross-section, plotted for various values of the stiffness parameter $\mathscr{K}$. If $\mathscr{F}<0(>0)$, the finger branches symmetrically (asymmetrically). The dashed lines represent the approximation $\mathscr{F}=2-\mathscr{K} / \mathrm{Ca}$ which provides excellent predictions for $\mathscr{F}(\mathrm{Ca})=0$. Results for tubes of rectangular, circular and elliptical cross-sections are qualitatively similar.

offer no resistance to the flow, we have $\mathscr{F}>0$, implying that the finger will branch asymmetrically.

\section{Comparing theory and experiment}

To compare theory and experiment, we must estimate the (volumetric) stiffness $k$ of the end chambers. As discussed in $\$ 2$, the end chambers are circular holes of radius $a=1.6 \mathrm{~mm}$ which are sealed with elastic membranes. Before the start of the experiment, the membranes were pre-stretched by injecting silicone oil until their centres were deflected outwards by approximately $\widehat{w} \approx 400 \mu \mathrm{m}$. Eschenauer $\&$ Schnell's (1986) large-displacement analysis of pressure-loaded circular membranes provides the relation between the chamber pressure and the membrane's maximum deflection, $p_{\text {elast }}(\widehat{w})$, and the corresponding chamber volume, $V(\widehat{w})$. Using these results we obtain the volumetric stiffness

$$
k=\frac{\mathrm{d} p_{\text {elast }}}{\mathrm{d} \widehat{w}}\left(\frac{\mathrm{d} V}{\mathrm{~d} \widehat{w}}\right)^{-1}=\frac{6 E h^{3}}{\pi a^{6}}\left(\frac{(23-9 v)}{7(1-v)}\left(\frac{\widehat{w}}{h}\right)^{2}+\frac{8}{3\left(1-v^{2}\right)}\right),
$$

which has a strong nonlinear dependence on $\widehat{w}$. The volume of PFD injected during the actual experiment is small relative to the total volume of the pressure chambers. Therefore, the injection of the PFD finger causes only small additional deflections and $k$ can be expected to remain approximately constant throughout the experiment. The flow resistance $\mathscr{R}$ of a rectangular channel of width $W$ and aspect ratio $2: 1$ is given by $\mathscr{R}=139.93 / W^{4}$ (see White 1991 ). Using the estimates for the physical parameters given in $\S 2$, we obtain $\mathscr{K}=k /(\mathscr{R} \sigma) \approx 1.0 \times 10^{-3}$, indicating that the transition to nonaxisymmetric branching should occur at a capillary number of $C a \approx \mathscr{K} / 2=5.0 \times 10^{-4}$. This compares favourably with the experimental data of figure 4 which shows that the transition between symmetric and asymmetric branching occurs for a finger 
velocity (in the parent branch) of $U \approx 2 \mathscr{U}$ between $478 \mu \mathrm{m} \mathrm{s}^{-1}$ and $606 \mu \mathrm{m} \mathrm{s}^{-1}$. This corresponds to capillary numbers (based on the velocities in the daughter branches) between $C a=\mu \mathscr{U} / \sigma=4.78 \times 10^{-4}$ and $6.06 \times 10^{-4}$.

\section{Discussion}

We have studied the propagation of low-viscosity fingers in a fluid-filled branching network of microchannels and have analysed the behaviour of the finger as it passes a single bifurcation. The behaviour was shown to depend mainly on the relative importance of viscous and elastic forces, characterized by the dimensionless parameter $\lambda=k /(2 \mu \mathscr{U} \mathscr{R})$. Viscous effects dominate if the finger velocity and/or the viscous flow resistance are large, so that $\lambda<1$. In this regime, the finger branches asymmetrically and will tend to open a single path through the network. This is because any perturbation that increases the length of one air finger relative to the length of the other, reduces the viscous flow resistance offered by the column of viscous fluid ahead of the finger tip. This causes the velocity of the longer finger to increase, enhancing the initial difference in finger length even further. We note that the 'inverse' of this mechanism is responsible for the symmetric propagation of the oil fingers that displace the air that initially fills the channel when the experiment is first set up. If a small local imperfection in the channel geometry momentarily allows one of the oil fingers to propagate slightly faster than the other, the resulting increase in the finger length increases the flow resistance and causes the finger to slow down, allowing the other finger to catch up.

Conversely, if the air finger propagates sufficiently slowly and/or if the stiffness of the end chambers is sufficiently large so that $\lambda>1$, the finger tends to branch symmetrically. This is because the slightly stronger inflation of the elastic end chamber that is connected to the branch conveying the longer finger, creates a strong restoring pressure which reduces the finger velocity until the volumes of both end chambers are approximately equal again.

In the context of the pulmonary airway reopening problem, our results suggest that the reopening of occluded airways should be performed at small speeds to encourage symmetric branching of the air finger as it propagates into the bronchial tree, though it is important to re-iterate that our model provides an extreme simplification of the conditions in the pulmonary airways. For instance, our model is based on a highly idealized geometry; our lumped representation of the wall elasticity ignores the fact that the wall deformation interacts with the fluid flow near the finger tip. Furthermore, while symmetric branching of the propagating air finger is clearly desirable, any attempt to optimize airway reopening procedures will be subject to many additional, and possibly conflicting, constraints. For instance, Bilek, Dee \& Gaver (2003) showed that airway reopening at small flow rates may result in cellular damage owing to an increase in the stresses on the airway wall. Finally, we assumed the finger to be driven by an imposed flow rate rather than a controlled driving pressure. In $\S 4$, we showed that $\mathrm{d} \widehat{p}_{d r} / \mathrm{d} t=0$, implying that there is no difference between the two cases, within the framework of a linear stability analysis. However, nonlinear effects are likely to become important when the deviations in the finger velocities become large. It is therefore conceivable that changes to the driving mechanism could affect the behaviour of the asymmetrically branching solution (see e.g. Halpern et al. 2005).

As for microfluidic flows, the results suggest that some limitations exist on the ability to fill a network of channels evenly or to divide drops. For this reason, it will be important to explore ways of stabilizing the symmetric branching in lab-on-a-chip 
applications, for example, by introducing an increased resistance to the flow as the finger advances, through the use of elastic forces.

M. H. was supported in part by an EGIDE visiting fellowship at Ecole Polytechnique. S. T. was supported in part by the MIT-France program. The authors acknowledge technical help from José Bico.

\section{REFERENCES}

Bilek, A. M, DeE, K. C. \& GaVer, D. P. 2003 Mechanisms of surface-tension-induced epithelial cell damage in a model of pulmonary airway reopening. J. Appl. Physiol. 94, 770-783.

Bretherton, F. P. 1961 The motion of long bubbles in tubes. J. Fluid Mech. 10, 166-188.

Calderon, A. J. \& Bull J. L. 2004 Homogeneity of bubble transport through a bifurcation for gas embolotherapy. FASEB J. 18, A373.

Cassidy, K. J., Gavriely, N. \& Grotberg, J. B. 2001 Liquid plug flow in straight and bifurcating tubes. J. Biomech. Engng 123, 580-589.

Eschenauer, H. \& Schnell, W. 1986 Elastizitätstheorie I. B. I.-Wissenschaftsverlag, Mannheim.

Gaver, D. P., Samsel, R. W. \& Solway, J. 1990 Effects of surface tension and viscosity on airway reopening. J. Appl. Physiol. 69, 74-85.

Halpern, D., Naire, S., Jensen, O. E. \& Gaver, D. P. III 2005 Unsteady bubble motion in a flexible-walled channel: predictions of a viscous stick-slip instability. J. Fluid Mech. 528, 53-86.

Hazel, A. L. \& Heil, M. 2003 Three-dimensional airway reopening: the steady propagation of a semi-infinite bubble into a buckled elastic tube. J. Fluid Mech. 478, 47-70.

Hazel, A. L. \& Heil, M. 2002 The steady propagation of a semi-infinite bubble into a tube of elliptical or rectangular cross-section. J. Fluid Mech. 470, 91-114.

Homsy, G. 1987 Viscous fingering in porous media. Annu Rev. Fluid Mech. 19, 271-311.

Link, D., Anna, S., Weitz, D. \& Stone, H. 2004 Geometrically mediated breakup of drops in microfluidic devices. Phys. Rev. Lett. 92, 054503.

QuAKe, S. R. \& SCHERER, A. 2000 From micro- to nano-fabrication with soft materials. Science 290, 1536-1540.

REINELT, D. \& SAFFMAN, P. 1985 The penetration of a finger into a viscous fluid in a channel and tube. SIAM J. Sci. Stat. Comput. 6, 542-561.

Song, H., Tice, J. \& Ismagilov, R. 2003 A microfluidic system for controlling reaction networks in time. Angew. Chem., Intl Edn 42.

TAYLOR, G. I. 1961 Deposition of a viscous fluid on the wall of a tube. J. Fluid Mech. 10, 161-165.

White, F. 1991 Viscous Fluid Flow. McGraw-Hill.

Wong, H., RadKe, C. J. \& Morris, S. 1995 The motion of long bubbles in polygonal capillaries. Part 1. Thin films. J. Fluid Mech. 292, 71-94. 